\title{
Environmental Risk Factors of Refractive Errors among Primary and Preparatory School Children in Assiut Governorate
}

\author{
Amal Ahmed Mobarak, Tarek Mohamed Aliy, Nahed Thabet Mohamed \& Asmaa Kamal Hassan. \\ Lecturer of Pediatric Nursing, Faculty of Nursing, Assiut University, Egypt. \\ Assistant Professor of Ophthalmology, Faculty of Medicine, Assiut University, Egypt. \\ Lecturer of Pediatric Nursing, Faculty of Nursing, Assiut University, Egypt. \\ Lecturer of Community Health Nursing, Faculty of Nursing Assuit University, Egypt.
}

\begin{abstract}
Refractive error (RE) is one of the most common problems of visual impairment, especially among school age children. Aim The study aimed to identify the environmental risk factors of refractive errors among primary and preparatory school children in Assiut Governorate. Subjects and Method: Descriptive cross sectional research design was used. The present study was conducted during the academic year 2012-2013 involving 12 urban and rural governmental schools. Three tools were used: Tool I: An interview questionnaire. Tool II: Socio-economic scale and Tool III: applied parallel visual screening test. Results: Female represents more than half of studied students $60.9 \%$ and $59.1 \%$ for students with RE and students without, respectively. The RE was significantly higher among students living in mixed residential areas (64.3\%) compared with the other residential areas. The RE was significantly higher among the group with RE exposed to near-work activity for more than 2hours / day compared with those exposed for less than 2 hours/day. Conclusion: The common risk factor of R E among primary and preparatory school children are source of pollution inside and outside home and students living in mixed residential areas have higher RE compared with the other residential areas. Recommendation: Development of national programme for early detection of visual impairment, involving both preschoolers and school age children, governmental support for providing low-cost spectacles and school support to encourage children to wear their glasses and early detection of environmental risk factors causes of poor vision, especially refractive errors.
\end{abstract}

Key words: Refractive Error, School Children, Near-Work/Day, Snellen Chart, Myopia. Hyperopia \& Astigmatism.

\section{Introduction}

A refractive error (RE) is a very common eye disorder occurs that when the eye cannot clearly focus the images from the outside world. Refractive errors result in blurred vision, which leads sometimes to visual impairment if it is so sever (Pascolini \& Mariotti, 2010 and WHO, 2013). Refractive errors are three main types namely; myopia, (near sightedness), hyperopia (far sightedness) and astigmatism. Myopia occurs when parallel light rays come to focus in front of the retina, due to too long anterior-posterior diameter of the eye. It may occur in children during the period of growth and development. The child usually complains of blurred vision for distant objects as difficulty in reading blackboard, writing in class room and perusing the distant activities. Near vision is usually not impaired except in high myopia. Myopia should be corrected with concave lenses (Pan et al., 2013 \& Datta, 2014).

Hyperopia occurs when the parallel light rays are focused behind the retina due to short anteroposterior diameter of the eye, poor refractive power of the cornea and dislocation of lens. The child is able to see distant objects clearly but near vision is impaired.
He may complain of eye strain, headache, redness of the eye, blurring of vision, fatigue and lid inflammation (Hockeberry \& Wilson, 2007 and Kyle, 2009). Astigmatism is a refractive error caused by the irregularity in the curvature of the cornea. It occurs, due to eye injury, or ptosis or hemangioma in eyelids. It is manifested as fatigue, squinting, headache, eye rubbing and eye strain. The child may be presented with poor performances and reading books (Datta, 2014).

Refractive error (RE) is the cause of a significant proportion of visual impairment and blindness. This problem has been recognized as a public health problem in many countries as well as the World Health Organization in its Vision 2020 initiative. RE is believed to result from a combination of genetic and environmental factors (Kyle, 2009). So the aim of the present study was to determine any factors associated with the occurrence of these refractive errors among the school age children (Rahi et al., 2010).

According to Resnikoff et al., (2008), Pascolini \& Mariotti (2010) it was reported that more than 12 million children aged 5-15 years old worldwide are 
visually impaired due to uncorrected refractive error and it is the leading cause of visual impairment worldwide.

In Egypt there are few studies showing the prevalence of errors of refraction. A survey conducted among 5839 Egyptian schoolchildren aged 7-15 years found that the prevalence of refractive errors (visual acuity $\leq 6 / 12$ ) was $22.1 \%$ (ElBayoumy et al., 2007). A preliminary national survey done in the Helwan area of Cairo reported that $34 \%$ of the recorded disabilities were visual disability (Shukrallah et al., 1997).

Refractive error is one of the most common problems of visual impairment, especially among school age children. It remains frequently undiagnosed for long periods. Approximately $20 \%$ of children have significant RE by their teen years (Vitale et al., 2006). Uncorrected refractive error is the main cause of moderate and severe visual impairment (VI) and the second leading cause of blindness (Resnikoff et al., 2008 \& Ayub et al., 2007). Visual impairment from uncorrected refractive errors can have immediate and long-term consequences in children and adults, such as lost educational and employment opportunities, lost economic gain for individuals, families and societies, and impaired quality of life (Rose et al., 2008 \& Ovenseri \& Assien, 2010). Various factors responsible for refractive errors remain uncorrected due to lack of awareness and recognition of the problem at the personal and the family level (Yingyong, 2010 \& Ayanniyi et al., 2010). These factors include positive history of wearing glasses in the family as well as close work or near activity such as prolonged study hours, watching computers/ television. On part of the community and public health level, non-availability to afford refractive services for testing, insufficient provision of affordable corrective lenses and cultural disincentives to compliance (Lempert, 2004).

The school age children suffering from ocular morbidity have huge physical, psychological and socio-economical implications. Early diagnosis of the ocular morbidity and correction help in the overall visual development and better academic performances as well (Morgan et al., 2012 \& Khalaj et al., 2009). The unilateral visual occlusion may be necessary and occasionally, surgery may be necessary. Support and reassurance according to the child development level are needed during the period of adjustment to contact lenses or eye glasses (Ricci \& Kyle, 2009).

Nursing management of the child with a RE focuses on providing education about corrective lenses use and monitoring the need for new eyeglasses or contact lenses. Nurse's role is to detect visual problems at an early age to prevent visual loss.
Provide health education for children about complete balanced diet and increase amount of vitamins, vegetables and fruits especially vitamin $\mathrm{A}$, encourage the child with newly eyeglasses to wear them continuously, instruct him and his family to clean glasses daily with mild soap and water, use a soft cloth to clean them not a paper towels, tissues or toilet paper. Teach the older child how to care for the contact lenses properly including lens hygiene insertion and removal. If the eye becomes inflamed, the contact lens showed be removed and wear eyeglasses until the eye improves (Datta, 2014).

\section{Significance of the study}

Vision is an important requirement for learning and communication. Further, optimal vision is essential for learning, health and educational needs (Zhao et al., 2004 \& Adegbehingbe, et al., 2005). School age children form especial group because they are most vulnerable to the effects of reduced vision and its impact on learning capability and educational potential. So that the objective of the present study was to evaluate preventable environmental risk factors for RE among these school children.

\section{Aim of the Study \\ The study aimed to identify the environmental risk factors of refractive errors among primary and preparatory school children in Assiut Governorate.}

\section{Subject \& Methods}

\section{Research Design}

Descriptive cross sectional research design was used. Research questions

What are the various environmental risk factors of refractive errors among primary and preparatory school children?

\section{Setting}

The present study was conducted at 12 urban and rural governmental schools in Assiut governorate: 4 primary mixed schools, 4 males preparatory schools and 4 female preparatory schools. The schools were chosen by multistage random sampling technique. The sites were selected to have different environmental characteristics, population activities and pollution sources namely; Elnazela and ElWaledia: Industrial and residential area in South east of Assuit governorate. Manqbad and asbat gouda: Mixed area (residential, industrial and heavy traffic) in North east Assiut governorate. New Assiut City: New residential area with traffic density low in South east of Assuit governorate.

Subject 
One thousand and four hundred fifty seven children from primary and preparatory schools in Assiut city. Children in Primary and preparatory schools were stratified into urban and rural governmental schools. A stratified random sampling was used to select 8 urban and 4 rural which near to the source of pollution from each stratum. Then, from each school, four classes were randomly selected from each of the academic years. Thus, a total of 396 classes from twelve schools were recruited, and the total sample size was 8660 students. The sample was divided into two groups; the student with RE $(n=657)$ and it was calculated $10 \%$ of the rest of the sample as a student without RE $(n=800)$. The student who suffer from any eye diseases was excluded from study.

Tools

Three tools were developed to conduct the study:

Tool I: An interview questionnaire: the tool was developed by the researchers to assess the risk factors of RE it is divided into four parts as follows:

Part (1): Socio-demographic characteristics of the students: which include name, age, sex, school level, and residential area.

Part (2): Medical data: which included ocular manifestations of eyestrain and previous ophthalmic problems such as, redness, headache and difficulty reading the blackboard.

Part (3): Environmental factors: about the residential area and sources of pollution. Such as sources of pollution near their houses: factories, workshops, rubbish dump and burn paper, burning brick kilns, mills and bakery. Cooking fuel used and the frequency of pesticides used by the mothers.

Part (4): Child performed near-work per day such as: reading, writing, watching television, playing on computer and play station.

Tool II: Socio-economic scale: This tool was developed by Abd El-Twaab (2004). to assess the socioeconomic status. It included four items namely; level of education of parents ( 8 items), family income (6 items), job of parent ( 2 items), life styles (3 items). Each item has one score the total score was classified into three classes as follows high class from 85$100 \%$, moderate class from 60 to less than $85 \%$ and low class less than $60 \%$. The item of income of social class was modified by the researchers as following; according to the rate of inflation and increase to be conforming with recent income through comparing difference of the value of the golden pound at 2004 to that at 2010 and multiplying the rate of inflation to the scale.

Tool III: Parallel visual screening

A Snellen chart is an eye chart used by eye care professionals and others to measure visual acuity. The Snellen chart is printed with eleven lines of block letters. The first line consists of one very large letter, which may be one of several letters, for example E, H, C or N. Subsequent rows have increasing numbers of letters that decrease in size. A person taking the test covers one eye from $20 \mathrm{ft}$. away, and reads aloud the letters of each row, beginning at the top. The smallest row that can be read accurately indicates the visual acuity in that eye. Instructions

- Explain the procedure to every child.

- Wash his/her hands as they will use a hand to cover one eye at a time.

- Test each eye separately - the affected eye first.

- Ensure good natural light or illumination on the chart.

Methods

- An official permission to carry out the study was obtained from vice minister of Education at Assuit governorate.

- The written consent from the manager of schools, oral agreement from the students and their parents was obtained after explaining the purpose and the nature of the study.

- Tool I was developed by the researcher and was tested for it is content, validity by five special nursing and ophthalmology experts.

- Reliability was estimated by Alpha Cronbach's test for the tool and its result was $\mathrm{R}=0.66$.

- A pilot study was carried out on $10 \%$ of the sample in selected setting to evaluate applicability and clarity of the tools. The sample was excluded from the study.

- The time taken to fill the sheets were took about $20-45$ minutes.

\section{First phase}

The first stage: all students ( 8660) were given structured questionnaire in each classroom in the selected schools, after that assembling the questionnaire from the students then apply parallel visual screening test and discover students who suffer from refractive errors the total number was 657.

\section{Second phase}

The healthy students were selected from list of the classrooms randomly and the total numbers were 8003 , the control group was chosen from the healthy students and their number was 800 students by ten percent. The students age ranged from 6-14 years and came from different socioeconomic areas. The collection of sampling took about 7 months in the first and second semester, through school visits in morning and afternoon shifts.

\section{Ethical Consideration}

Ethical approval was obtained from the relevant research ethical committee in the Faculty of Nursing, Assiut University, to approve the research. Oral 
consent was obtained from all the students' to participate in the research after explaining the aim of the study, its benefits, duration of the study, and data collection tool. The participants' parents were assured that the researcher investigated the students and identify the students with refractive errors by using Sellen chart.

Risk-benefit assessment. There is no risk for students' at all during application of the research and all mothers were reassured that information obtained well be confidential and used only for the purpose of the study.

\section{Statistical Design}

Categorical variables were described by number and percent (N, \%), where continuous variables described by mean and standard deviation (Mean, SD). Chi-square test used to compare between categorical variables where compare between continuous variables by t-test and ANOVA. Continuous variables were tested for normal distribution using Kolmogorove Smirnov test and Q-Q Plots. A two-tailed $\mathrm{p}<0.05$ was considered statistically significant. All analyses were performed with the IBM SPSS 20.0 software.

\section{Results}

Table (1): Percentages distribution of students according to their socio demographic characteristics.

\begin{tabular}{|c|c|c|c|c|c|c|}
\hline \multirow[t]{2}{*}{ Items } & \multicolumn{2}{|c|}{$\begin{array}{c}\text { Students with RE } \\
(\mathrm{n}=657)\end{array}$} & \multicolumn{2}{|c|}{$\begin{array}{c}\text { Students without RE } \\
(\mathrm{n}=\mathbf{8 0 0})\end{array}$} & \multirow[t]{2}{*}{$\mathbf{X}^{2}$} & \multirow[t]{2}{*}{ p. value } \\
\hline & No. & $\%$ & No. & $\%$ & & \\
\hline \multicolumn{7}{|l|}{ Age: in years } \\
\hline $6-12$ & 369 & 56.2 & 480 & 60.0 & \multirow[t]{2}{*}{2.18} & \multirow[t]{2}{*}{0.139} \\
\hline$>12-15$ & 288 & 43.8 & 320 & 40.0 & & \\
\hline mean \pm SD & \multicolumn{2}{|c|}{$11.2 \pm 2.3$} & \multicolumn{2}{|c|}{$11.4 \pm 2.5$} & $t=1.57$ & 0.115 \\
\hline \multicolumn{7}{|l|}{ Gender: } \\
\hline Male & 257 & 39.1 & 327 & 40.9 & \multirow[t]{2}{*}{0.46} & \multirow[t]{2}{*}{0.496} \\
\hline Female & 400 & 60.9 & 473 & 59.1 & & \\
\hline \multicolumn{7}{|l|}{ Level of education } \\
\hline Primary & 327 & 49.8 & 430 & 53.8 & \multirow[t]{2}{*}{2.29} & \multirow[t]{2}{*}{0.130} \\
\hline Preparatory & 330 & 50.2 & 370 & 46.3 & & \\
\hline \multicolumn{7}{|l|}{ Residence } \\
\hline Rural & 158 & 24 & 220 & 27.5 & \multirow[t]{2}{*}{2.24} & \multirow[t]{2}{*}{0.135} \\
\hline Urban & 499 & 76 & 580 & 72.5 & & \\
\hline \multicolumn{7}{|c|}{ Socio- economic class } \\
\hline Low & 138 & 21.0 & 165 & 20.6 & \multirow[t]{3}{*}{4.81} & \multirow[t]{3}{*}{0.090} \\
\hline Middle & 435 & 66.2 & 500 & 62.5 & & \\
\hline High & 84 & 12.8 & 135 & 16.9 & & \\
\hline
\end{tabular}

Chi-square test used for comparison in this table.

${ }^{n}:$ No statistically significant difference $(p>0.05)$

Independent t-test used for comparing age

Table (2): Percentages distribution of students according to the sources of pollution out-side and inside home.

\begin{tabular}{|c|c|c|c|c|c|c|}
\hline \multirow{2}{*}{ Items } & \multicolumn{2}{|c|}{$\begin{array}{l}\text { Students with RE } \\
\qquad(\mathrm{n}=657)\end{array}$} & \multicolumn{2}{|c|}{$\begin{array}{l}\text { Students without } \\
\text { RE }(\mathbf{n}=\mathbf{8 0 0})\end{array}$} & \multirow[t]{2}{*}{$\mathbf{X}^{2}$} & \multirow[t]{2}{*}{ P. value } \\
\hline & Yes & $\%$ & No & $\%$ & & \\
\hline \multicolumn{7}{|l|}{ Source of pollutions out-side home:\# } \\
\hline Factories & 251 & 38.2 & 150 & 18.8 & 68.4 & $<0.001 * *$ \\
\hline Workshops & 355 & 54.0 & 300 & 37.5 & 39.8 & $<0.001 * *$ \\
\hline Rubbish dump and burn paper & 406 & 61.8 & 320 & 40.0 & 68.5 & $<0.001 * *$ \\
\hline Burning brick kilns & 202 & 30.7 & 126 & 15.8 & 46.5 & $<0.001 * *$ \\
\hline Mills & 185 & 28.2 & 107 & 13.4 & 49.2 & $<0.001 * *$ \\
\hline
\end{tabular}




\begin{tabular}{|c|c|c|c|c|c|c|}
\hline \multirow{2}{*}{ Items } & \multicolumn{2}{|c|}{$\begin{array}{c}\text { Students with RE } \\
(n=657)\end{array}$} & \multicolumn{2}{|c|}{$\begin{array}{l}\text { Students without } \\
\text { RE }(\mathbf{n}=\mathbf{8 0 0})\end{array}$} & \multirow[t]{2}{*}{$\mathbf{X}^{2}$} & \multirow[t]{2}{*}{ P. value } \\
\hline & Yes & $\%$ & No & $\%$ & & \\
\hline Bakery & 507 & 77.2 & 375 & 46.9 & 138.6 & $<0.001 * *$ \\
\hline \multicolumn{7}{|c|}{ Source of pollutions inside home:\# } \\
\hline Cooker & 383 & 58.3 & 311 & 38.9 & 54.5 & $<0.001 * *$ \\
\hline Natural gas & 198 & 30.1 & 230 & 28.8 & 0.33 & 0.603 \\
\hline Kerosene and canon & 76 & 11.6 & 105 & 13.1 & 0.8 & 0.414 \\
\hline Sprayed insecticides & 400 & 60.9 & 520 & 65.0 & 2.63 & 0.117 \\
\hline
\end{tabular}

** Statistically significant difference (p level <0.01)

(\#) more than one answer is available

Table (3): Percentages distribution of students according to their performed near work per day.

\begin{tabular}{|c|c|c|c|c|c|c|}
\hline \multirow{2}{*}{$\begin{array}{c}\text { Group } \\
\text { Near-work } \\
\text { per day }\end{array}$} & \multicolumn{2}{|c|}{$\begin{array}{c}\text { Students with RE } \\
(n=657)\end{array}$} & \multicolumn{2}{|c|}{$\begin{array}{c}\text { Students without } \\
\text { RE }(\mathbf{n}=\mathbf{8 0 0})\end{array}$} & \multirow[t]{2}{*}{ Chi - Square } & \multirow[t]{2}{*}{ p. value } \\
\hline & No. & $\%$ & No. & $\%$ & & \\
\hline Reading & 657 & 100.0 & 800 & 100.0 & & \\
\hline 1-2 hrs/day & 157 & 23.9 & 550 & 68.7 & \multirow{2}{*}{290.5} & \multirow{2}{*}{$<0.001 * *$} \\
\hline More than 2 hrs/day & 500 & 76.1 & 250 & 31.3 & & \\
\hline $\bar{\chi} \pm$ SD & \multicolumn{2}{|c|}{$2.8 \pm 1.2$} & \multicolumn{2}{|c|}{$1.8 \pm 0.7$} & $\mathrm{t}=19.8$ & $<0.001 * *$ \\
\hline Writing & 657 & 100.0 & 800 & 100.0 & & \\
\hline 1-2 hrs/day & 224 & 34.1 & 620 & 77.5 & \multirow{2}{*}{278.8} & \multirow{2}{*}{$<0.001 * *$} \\
\hline More than 2 hrs/day & 433 & 65.9 & 180 & 22.5 & & \\
\hline $\bar{\chi} \pm$ SD & \multicolumn{2}{|c|}{$1.9 \pm 0.8$} & \multicolumn{2}{|c|}{$1.6 \pm 0.3$} & $\mathrm{t}=9.8$ & $<0.001 * *$ \\
\hline Watching TV & 623 & 94.8 & 800 & 100.0 & & \\
\hline 1-2 hrs/day & 222 & 35.6 & 90 & 88.7 & \multirow{2}{*}{121.6} & \multirow{2}{*}{$<0.001 * *$} \\
\hline More than 2 hrs/day & 401 & 64.4 & 710 & 11.3 & & \\
\hline $\bar{\chi} \pm \mathbf{S D}$ & \multicolumn{2}{|c|}{$1.7 \pm 0.7$} & \multicolumn{2}{|c|}{$2.4 \pm 0.9$} & $t=16.3$ & $<0.001 * *$ \\
\hline Not watching TV & 34 & 5.2 & 0 & 0.0 & & \\
\hline Using the computer & 402 & 61.2 & 800 & 100.0 & & \\
\hline 1-2 hrs/day & 100 & 24.9 & 125 & 84.4 & \multirow{2}{*}{15.1} & \multirow{2}{*}{$<0.001 * *$} \\
\hline More than 2 hrs/day & 302 & 75.1 & 675 & 15.6 & & \\
\hline $\bar{x} \pm$ SD & \multicolumn{2}{|c|}{$1.7 \pm 0.5$} & \multicolumn{2}{|c|}{$2.3 \pm 1.1$} & $\mathrm{t}=12.9$ & $<0.001 * *$ \\
\hline Not using the computer & 255 & 38.8 & $\mathbf{0}$ & $\mathbf{0 . 0}$ & & \\
\hline Using play station & 402 & 61.2 & 800 & 100.0 & & \\
\hline 1-2 hrs/day & 95 & 23.6 & 111 & 86.1 & \multirow{2}{*}{17.9} & \multirow{2}{*}{$<0.001 * *$} \\
\hline More than 2 hrs/day & 307 & 76.4 & 689 & 13.9 & & \\
\hline $\bar{\chi} \pm$ SD & \multicolumn{2}{|c|}{$1.8 \pm 0.6$} & \multicolumn{2}{|c|}{$2.4 \pm 1.3$} & $\mathrm{t}=10.9$ & $<0.001 * *$ \\
\hline Not using play station & 255 & 38.8 & 0 & 0.0 & & \\
\hline
\end{tabular}

** Statistically significant difference (p level <0.01) 
Table (4): Relations between measuring sight and near-work per day in studied children with refractive errors.

\begin{tabular}{|c|c|c|c|c|c|c|c|c|c|c|c|c|c|c|}
\hline \multirow{3}{*}{$\begin{array}{c}\text { Measuring } \\
\text { sight } \\
\text { Near-work } \\
\text { per day } \\
\end{array}$} & \multicolumn{6}{|c|}{ Right eye } & \multirow{3}{*}{$\begin{array}{c}\text { P. } \\
\text { value }\end{array}$} & \multicolumn{6}{|c|}{ Left eye } & \multirow{3}{*}{$\begin{array}{c}P . \\
\text { value }\end{array}$} \\
\hline & \multicolumn{2}{|c|}{$\begin{array}{c}\text { Mild low } \\
\text { vision } \\
(\mathbf{n}=131)\end{array}$} & \multicolumn{2}{|c|}{$\begin{array}{c}\text { Moderate } \\
\text { low vision } \\
(\mathbf{n}=\mathbf{2 4 1})\end{array}$} & \multicolumn{2}{|c|}{$\begin{array}{c}\text { Severe low } \\
\text { vision } \\
(\mathrm{n}=247)\end{array}$} & & \multicolumn{2}{|c|}{$\begin{array}{c}\text { Mild vision } \\
(\mathrm{n}=181)\end{array}$} & \multicolumn{2}{|c|}{$\begin{array}{c}\text { Moderate } \\
\text { low vision } \\
(\mathbf{n}=\mathbf{2 7 5})\end{array}$} & \multicolumn{2}{|c|}{$\begin{array}{c}\text { Severe low } \\
\text { vision } \\
(\mathbf{n}=186)\end{array}$} & \\
\hline & No. & $\%$ & No. & $\%$ & No. & $\%$ & & No. & $\%$ & No. & $\%$ & No. & $\%$ & \\
\hline Reading: & 131 & 100 & 241 & 100 & 247 & 100 & & 181 & 100 & 275 & 100 & 186 & 100 & \\
\hline 1-2 hrs/day & 33 & 25.2 & 66 & 27.4 & 50 & 20.2 & \multirow{2}{*}{$\begin{array}{c}\mathrm{X}^{2}=3.5 \\
0.172\end{array}$} & 30 & 16.6 & 75 & 27.3 & 45 & 24.2 & \multirow{2}{*}{$\begin{array}{l}X^{2}=7.1 \\
0.029 *\end{array}$} \\
\hline More than 2 hrs/day & 98 & 74.8 & 175 & 72.6 & 197 & 79.8 & & 151 & 83.4 & 200 & 72.7 & 141 & 75.8 & \\
\hline $\bar{x} \pm \mathbf{S D}$ & \multicolumn{2}{|c|}{$2.05 \pm 0.87$} & \multicolumn{2}{|c|}{$1.91 \pm 0.68$} & \multicolumn{2}{|c|}{$2.07 \pm 0.8$} & $\begin{array}{l}\mathrm{F}=2.1 \\
0.041 *\end{array}$ & \multicolumn{2}{|c|}{$2.14 \pm 0.81$} & \multicolumn{2}{|c|}{$1.94 \pm 0.73$} & \multicolumn{2}{|c|}{$1.99 \pm 0.78$} & $\begin{array}{l}\mathrm{F}=2.3 \\
0.020 *\end{array}$ \\
\hline Writing: & 131 & 100 & 241 & 100 & 247 & 100 & & 181 & 100 & 275 & 100 & 186 & 100 & \\
\hline 1-2 hrs/day & 46 & 35.1 & 90 & 37.3 & 78 & 31.6 & \multirow{2}{*}{$\begin{array}{c}X^{2}=1.8 \\
0.404\end{array}$} & 57 & 31.5 & 95 & 34.5 & 65 & 34.9 & \multirow{2}{*}{$\begin{array}{c}\mathrm{X}^{2}=0.6 \\
0.738\end{array}$} \\
\hline More than 2 hrs/day & 85 & 64.9 & 151 & 62.7 & 169 & 68.4 & & 124 & 68.5 & 180 & 65.5 & 121 & 65.1 & \\
\hline $\bar{x} \pm$ SD & \multicolumn{2}{|c|}{$1.85 \pm 0.79$} & \multicolumn{2}{|c|}{$1.93 \pm 0.9$} & \multicolumn{2}{|c|}{$1.93 \pm 0.82$} & $\begin{array}{l}F=0.9 \\
0.665\end{array}$ & \multicolumn{2}{|c|}{$1.95 \pm 0.82$} & \multicolumn{2}{|c|}{$1.93 \pm 0.85$} & \multicolumn{2}{|c|}{$1.9 \pm 0.84$} & $\begin{array}{l}\mathrm{F}=0.7 \\
0.872\end{array}$ \\
\hline Watching TV: & 121 & 92.4 & 228 & 94.6 & 236 & 95.5 & & $\begin{array}{c}17 \\
7\end{array}$ & 92.4 & 254 & 92.4 & 177 & 95.2 & \\
\hline $1-2 \mathrm{hrs} /$ day & 41 & 31.3 & 84 & 34.9 & 83 & 33.6 & \multirow{2}{*}{$\begin{array}{c}X^{2}=0.3 \\
0.849\end{array}$} & 67 & 37.0 & 86 & 31.3 & 62 & 33.3 & \multirow{2}{*}{$\begin{array}{c}X^{2}=0.7 \\
0.691\end{array}$} \\
\hline More than 2 hrs/day & 80 & 61.1 & 144 & 59.8 & 153 & 61.9 & & $\begin{array}{c}11 \\
0\end{array}$ & 60.8 & 168 & 61.1 & 115 & 61.8 & \\
\hline $\bar{x} \pm$ SD & 1.6 & 0.52 & 1.7 & .56 & 1.7 & 0.55 & $\begin{array}{l}F=0.8 \\
0.748\end{array}$ & & $\mathbf{0 . 5 7}$ & 1.7 & 0.53 & 1.7 & 0.54 & $\begin{array}{c}F=1.4 \\
0.320\end{array}$ \\
\hline No watching TV: & 10 & 7.6 & 13 & 5.4 & 11 & 4.5 & & 4 & 2.2 & 21 & 7.6 & 9 & 4.8 & \\
\hline Using computer: & 99 & 75.6 & 133 & 55.2 & 139 & 56.3 & & $\begin{array}{c}13 \\
5\end{array}$ & 92.4 & 164 & 59.6 & 94 & 50.5 & \\
\hline 1-2 hrs/day & 27 & 20.6 & 32 & 13.3 & 31 & 12.6 & $\mathrm{X}^{2}=0.8$ & 42 & 23.2 & 37 & 13.5 & 18 & 9.7 & $\mathrm{X}^{2}=4.9$ \\
\hline More than 2 hrs/day & 72 & 55.0 & 101 & 41.9 & 108 & 43.7 & & 93 & 51.4 & 127 & 46.2 & 76 & 40.9 & \\
\hline $\bar{x} \pm \mathbf{S D}$ & 1.6 & .52 & & .56 & 1.7 & .55 & $\begin{array}{l}\mathrm{F}=0.8 \\
0.748\end{array}$ & & $\mathbf{0 . 5 7}$ & 1.7 & 0.53 & 1.7 & 0.54 & $\begin{array}{l}F=1.4 \\
0.320\end{array}$ \\
\hline No using computer: & 32 & 24.4 & 108 & 44.8 & 108 & 43.7 & & 46 & 25.4 & 111 & 40.4 & 92 & 49.5 & \\
\hline Using play station: & 99 & 75.6 & 133 & 55.2 & 139 & 56.3 & & $\begin{array}{c}13 \\
5\end{array}$ & 92.4 & 164 & 59.6 & 94 & 50.5 & \\
\hline 1-2 hrs/day & 26 & 19.8 & 32 & 13.3 & 29 & 11.7 & $\mathrm{X}^{2}=$ & 37 & 20.4 & 38 & 13.8 & 18 & 9.7 & $\mathrm{X}^{2}=2.1$ \\
\hline More than 2 hrs/day & 73 & 55.7 & 101 & 41.9 & 110 & 44.5 & & 98 & 54.1 & 126 & 45.8 & 76 & 40.9 & 0.3 \\
\hline $\bar{x} \pm$ SD & 1.7 & 0.58 & 1. & 0.62 & 1. & 0.66 & $\begin{array}{l}\mathrm{F}=1.1 \\
0.593 \\
\end{array}$ & & $\mathbf{0} 0.58$ & 1.7 & 0.64 & 1.7 & 0.65 & $\begin{array}{l}F=0.6 \\
0.855\end{array}$ \\
\hline $\begin{array}{l}\text { No using play } \\
\text { station }\end{array}$ & 32 & 24.4 & 108 & 44.8 & 108 & 43.7 & & 46 & 25.4 & 111 & 40.4 & 92 & 49.5 & \\
\hline
\end{tabular}

* Statistically significant difference (p level <0.05)

Table (5): Relation between age groups and near-work activity per day of studied children with refractive errors.

\begin{tabular}{|c|c|c|c|c|c|c|}
\hline \multirow{2}{*}{ Near-work activity per day } & \multicolumn{2}{|c|}{$\begin{array}{c}\text { 6-12 years } \\
(n=369)\end{array}$} & \multicolumn{2}{|c|}{$\begin{array}{c}>12-15 \text { years } \\
(n=288)\end{array}$} & \multirow[t]{2}{*}{$X^{2}$} & \multirow[t]{2}{*}{ P. value } \\
\hline & No. & $\%$ & No. & $\%$ & & \\
\hline Reading & 369 & 100.0 & 288 & 100.0 & & \\
\hline 1-2 hrs/day & 71 & 19.2 & 86 & 29.9 & \multirow{2}{*}{10.0} & \multirow{2}{*}{$0.002 * *$} \\
\hline More than 2 hrs/day & 298 & 80.8 & 202 & 70.1 & & \\
\hline Writing & 369 & 100.0 & 288 & 100.0 & & \\
\hline 1-2 hrs/day & 126 & 34.1 & 98 & 34.0 & \multirow{2}{*}{0.001} & \multirow{2}{*}{0.978} \\
\hline More than 2 hrs/day & 243 & 65.9 & 190 & 66.0 & & \\
\hline
\end{tabular}




\begin{tabular}{|c|c|c|c|c|c|c|}
\hline \multirow{2}{*}{ Near-work activity per day } & \multicolumn{2}{|c|}{$\begin{array}{c}\text { 6-12 years } \\
(n=369)\end{array}$} & \multicolumn{2}{|c|}{$\begin{array}{c}12-15 \text { years } \\
(n=288)\end{array}$} & \multirow[t]{2}{*}{$\mathrm{X}^{2}$} & \multirow[t]{2}{*}{ P. value } \\
\hline & No. & $\%$ & No. & $\%$ & & \\
\hline Watching TV & 369 & 100.0 & 254 & 88.2 & & \\
\hline $1-2 \mathrm{hrs} /$ day & 160 & 43.4 & 62 & 21.5 & \multirow{2}{*}{23.6} & \multirow{2}{*}{$0.001 * *$} \\
\hline More than 2 hrs/day & 209 & 56.6 & 192 & 66.7 & & \\
\hline No watching $T V$ & 0 & 0.0 & 34 & 11.8 & & \\
\hline Using computer & 229 & 62.1 & 173 & 60.1 & & \\
\hline $1-2$ hrs/day & 80 & 21.7 & 20 & 6.9 & \multirow{2}{*}{28.8} & \multirow{2}{*}{$0.001 * *$} \\
\hline More than 2 hrs/day & 149 & 40.4 & 153 & 53.1 & & \\
\hline No using computer & 140 & 37.9 & 115 & 39.9 & & \\
\hline Using play station & 229 & 62.1 & 173 & 60.1 & & \\
\hline $1-2$ hrs/day & 72 & 19.5 & 23 & 8.0 & \multirow{2}{*}{18.0} & \multirow{2}{*}{$0.001 * *$} \\
\hline More than 2 hrs/day & 157 & 42.5 & 150 & 52.1 & & \\
\hline No using play station & 140 & 37.9 & 115 & 39.9 & & \\
\hline
\end{tabular}

** Statistically significant difference (p level <0.01)

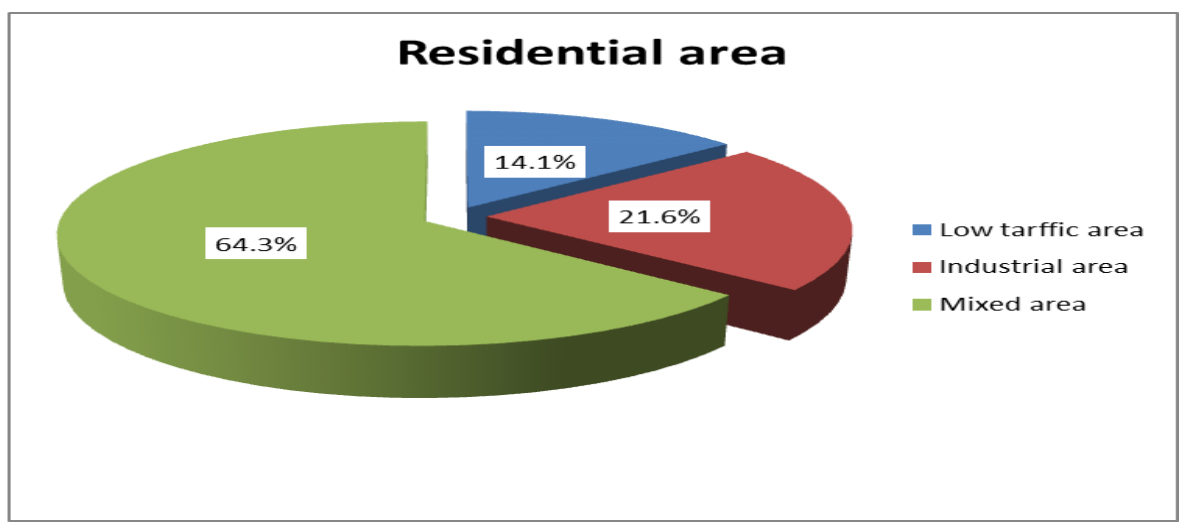

Figure(1): Percentage distribution of children with refractive error regarding type of residential area.

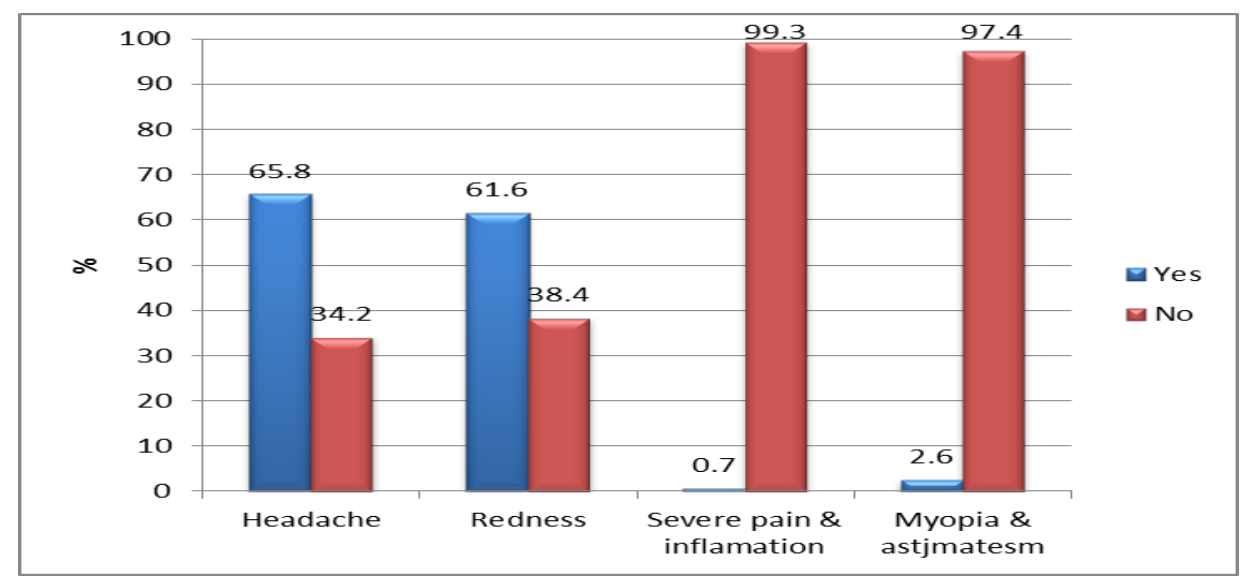

Figure (2): Percentage distribution of children with refractive error regarding signs and symptoms. 


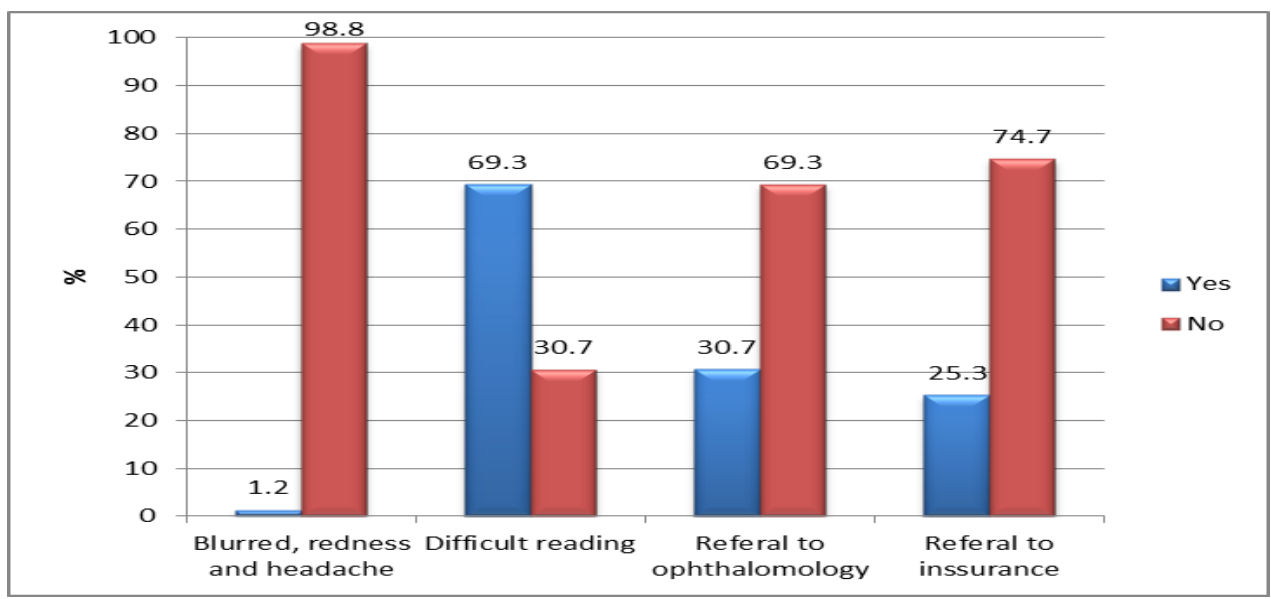

Figure (3): Percentage distribution of children with refractive error related to present cause and site.

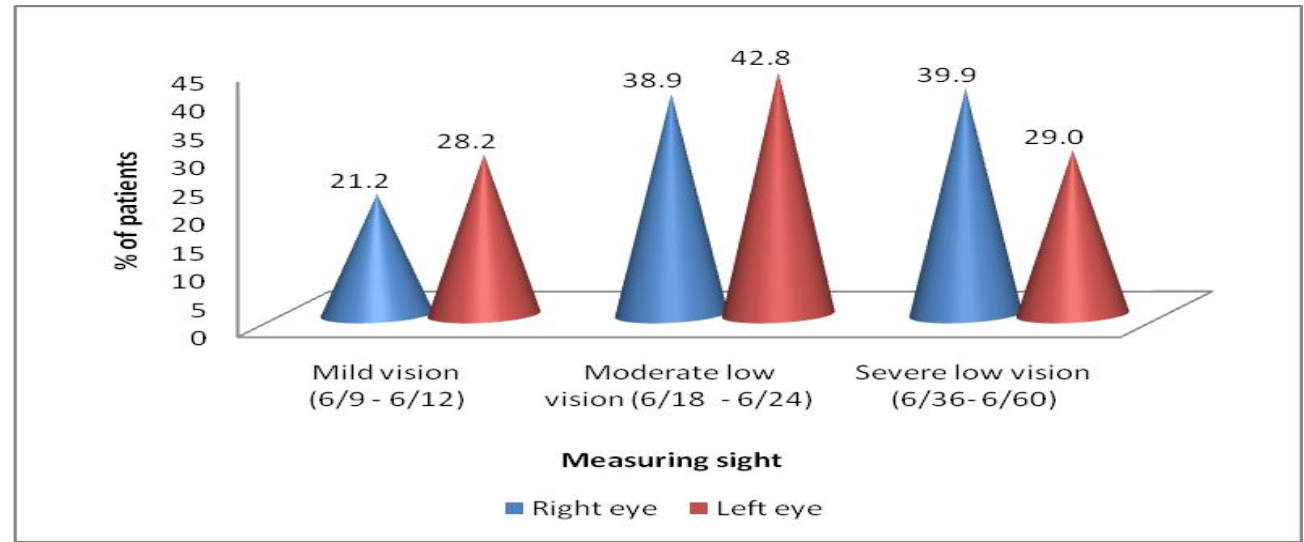

Figure (4): Percentage distribution of children with refractive error regarding to measuring sight (Right \& Left eyes).

Table (1): Represents percentage distribution of socio demographic characteristics with mean age $11.2 \pm 2.3$ years and $11.4 \pm 2.5$ years for, respectively. Female represents more than half of students with RE $60.9 \%$ and $59.1 \%$ for students without, respectively.

Near half of students with RE in the had primary education compared to slightly higher than half of them in the students without. As regards residence the majority of studied students came from urban areas $(76 \%$ and $72.5 \%$ for the students with RE and students without, respectively. Regarding socioeconomic class the higher percentage of students had middle socio-economic class with no significant differences between students with RE and students without.

Table (2): This table revealed that the students with RE were from different residential areas with different environmental conditions. More than three quarters present in area near bakery, $61.8 \%$ of them near rubbish dump and burn paper compared to $46.9 \%$ and $40.0 \%$ respectively in the students without. More than half to $38.9 \%$ in the control group. More than half $(60.9 \%)$ of the students with $\mathrm{RE}$ and students without whose mother constant sprayed insecticides in home were suffering from RE compared to $65 \%$ in the students without. Statistically significant differences were found between the two groups regarding to all items except for natural gas, kerosene and canon and sprayed insecticides.

Table (3): This table shows that $76.1 \%$ and $65.9 \%$ of students with RE were able to read and write more than $2 \mathrm{hrs} /$ day compared to $31.3 \%$ and $22.5 \%$ of the students without respectively. The results indicated that the prevalence of RE was significantly higher among students with RE exposed to near-work for more than 2hours / day compared with those exposed for less than 2 hours / day such as watching TV, using computer and play station. Statistically 
significant differences were found between the two groups regarding to all items.

Table (4): This table shows the relationship between measuring sight and near-work activity per day hours. Seventy nine point eight $79.8 \%$ and $68.4 \%$ of students whose read and write for more than 2 hours had severe low vision problem in the right eye. While $83.4 \%$ and $68.5 \%$ of them had mild vision problem in the left eye. As regards to watching TV for more than 2 hrs, more than two thirds of the students $(61.9 \%$ and $61.8 \%$ ), respectively had severe low vision in both eyes. The results revealed that, more than half who using computer and play station for more than 2 hours had mild vision problem in the right and left eye.

Table (5): This table shows relationship between hours of near work activity per/ day and child age. The percent of students whose age less than 12 years and were writing, watching TV, using computer and play station for more than two hours were high when compared with the students aged from 6-12 years but the percent of students aged from 6-12 years was high related to reading $(80.8 \%)$ when compared with the old age students $(70.1 \%)$.

Figure (1): shows the Percentage distribution of children with refractive error regarding type of residential area was significantly higher among students living in mixed residential areas (64.3\%) compared with the other residential areas, low traffic area $(14.1 \%)$ and industrial area $(21.6 \%)$.

Figure (2): shows some symptoms and signs of ocular problems in the students with refractive error in the form of eyestrain as headache $(65.8 \%)$, redness in the eyes $(61.6 \%),(0.7 \%)$ severe pain and inflammation and $(2.6 \%)$ of students with RE present with myopia and astigmatism.

Figure (3): This figure shows that $69.3 \%$ referred due to difficult reading, $30.7 \%$ referred to ophthalmologist, $25.3 \%$ of children referred to health insurance and finally $1.2 \%$ referred due to presence of blurred vision, redness in the eyes and headache.

Figure (4): This figure shows that measuring sight (Right \& Left eyes). Where it was clear that mild low vision (21.2\% and $28.2 \%)$, moderate low vision $(38.9 \%$ and $42.8 \%)$, severe low vision $(39.9 \%$ and $29.0 \%$ ) of studied students.

\section{Discussion}

School age children are considered one of the most important sectors of population due to their continuous growth and development at all levels. They are a vulnerable group and great attention should be paid for them (Bataineh \& Khatatbeh, 2008). So, coordinated school health programs in conjunction with community efforts can prevent many health problems among students and help them to establish lifelong safety skills (Allensworth et al., 1997 \& El-Moselhy et al., 2005a).

More than half of children were in the age group of 6-12years. While $43.8 \%$ aged from 12 years or more. This result disagree with many authors as El Bayoumy \& Saad (2007) who found that the prevalence of RE was significantly higher among students aged 12-15 years compared with those below 12 years; Pavithra et al., (2013) who found that, the prevalence of refractive error was more $(7.5 \%)$ in the $13-15$ years age group compared to $6.6 \%$ of 7-9 years age group; Matta et al (2005), who found that refractive error increased with increasing age especially in the age group of 10-14 years and Khandekar \& Abdu-Helmi (2004) showed that rate of vision impairment was significantly higher among high age group. Results of the present study are in the same line with Baltussen et al., (2008) reported that the incidence of refractive error is most frequent at 8-10 years of age.

In the present study more than thirty percent $(39.1 \%)$ of the children were males. While $60.9 \%$ were females. These results disagreement with Shrestha et al., (2009) who reported that, male sex was significant risk factor. Also contrary to Katz et al., (1997) who found that no sex difference related to RE. Finding of the present study are similar to El Bayoumy \& Saad (2007) who found that the prevalence of RE was higher among female students compared to males. Also Adegbehingbe et al., (2005) \& El-Moselhy et al., (2005b) found that female sex was risk factor for vision impairment. Pavithra et al., (2013) reported that Refractive error was more prevalent in the female children (9\%) compared to male children $(5.3 \%)$. Sharma et al., (2009) found that prevalence of refractive error was $23.7 \%$ in girls and only $12.2 \%$ in boys. Similar results were found by Tay et al., (1992) in their study on young Singaporeans. They related this high prevalence to the higher rate of growth in girls and also because girls attain puberty earlier than boys. Wong, (2007) attributed the elevation of RE among females to the fact that women's eyes have a shorter axial length and shallower anterior chamber depth than those of males.

Regarding the educational level, in the present study more than half of children $(50.2 \%)$ were in the preparatory school,(49.8\%) were in primary school. This results agree with El Bayoumy \& Saad (2007) who found that, the prevalence of RE was slightly higher among preparatory school students than among primary students. Also Afghani et al., (2003) who reported that prevalence of refractive errors are now linked to increase in academic levels. Ali et al., (2007), \& Hashim et al. (2008), who found that the 
prevalence of refractive errors in higher grade level (5-8) was about 4.8 times more likely than lower grade (1-4)

Most of children in the sample were from urban areas in Assiut and the rest of them were from rural areas.

Dandona et al., (2002), \& Murthy et al., (2002), who reported that, the effect of urbanization in refractive error has been reported in studies conducted in India and they have shown that, the prevalence of myopia and hyperopia in urban India was higher than in rural India. They observe the similar trend in refractive error prevalence in Kathmandu $(8.1 \%)$ in an urbanized area and $(3 \%)$ in a rural location. Also, Pavithra et al., (2013), who found that, the prevalence of refractive error was significantly high $(\mathrm{P}=0.0249)$ in the urban area, similar to the findings of Vivek \& Trivedi et al., (2008) in their population based study in Gujarat. Padhye et al., (2010), also reported a higher prevalence of $5.46 \%$ among the children in the urban area compared to $2.63 \%$ in rural India. It found that the rate of refraction errors increase in the urban area compare to rural areas, it may be explained that the presence of factories and garbage in the urban area.

As regards socioeconomic status of families of studied children, indicated that, the prevalence of RE was higher in the middle status. The results inconsistence with Dandona, (2001) who stated that children with higher and lower socioeconomic status were more likely to have RE than those of middle status. Also, Essam et al., (2011), who mentioned that, the low level of parental education, occupation and low social level were significant risk factors for eye diseases. On the other hand, the high level of parental education, occupation and high social level were significant protective factors for eye diseases. This results may be explained by students of middle socioeconomic status may spend a longer time more than 2 hours each day in reading, writing, watching television, playing computer games and Atari. It has been hypothesized that prolonged reading or the retinal blur of prolonged near-work leads to the development of myopia. The present data revealed that socioeconomic status was found to be the most important environmental factor that might affect an individual's risk of developing RE.

Past history of ocular problems was found in the students with RE in the form of symptoms of eyestrain as redness in the eye, headache, severe pain and inflammation, myopia and astigmatism. These results are consistence with Beers \& Porter, (2006) who stated that refractive errors principally result in visual blurriness at short or long distance. In some cases headache may also occur. Occasionally, the eye surface may become dry, resulting in eye irritation, itching, visual fatigue, the sensation of a foreign body, or redness.

Regarding to cause and site of referral, in this result high percentage of students referred due to difficult reading on blackboard, one third referred to ophthalmologist, small number of students referred to health insurance and finally low percent due to blurred vision, redness in the eyes and headache. Our results are consistence with El Bayoumy \& Saad, (2007) who stated that, the students referred in the following condition as redness of the eyes, headache, difficulty reading in blackboard in class or recorded comments in the medical file of the child about referral to ophthalmologists. The sing and symptom which appear on the student may be explained by the presence of students for long periods inside the school and the school toilet was not contain soap for washing their hands after feeding or playing and also the school yard was not prepared well, exposing them to dust.

The results indicated that the prevalence of RE was significantly higher among students exposed to nearwork activity for more than 2 hours / day compared with those exposed for less than 2 hours / day such as reading, writing, watching $\mathrm{TV}$, using computer and play station. The results similar to Mutti et al., \& Saw et al., (2002), who found that, the refractive errors have been associated with certain factors e.g. a positive history of wearing glasses in the family, close work or near activity such as prolonged study hours, watching computers / television. Also this finding supported by Alam et al., (2008), who showed that using of computers regularly had increased the chance of having refractive error by $4.5 \%$ compared with irregular or non-users. This could be due to the effect of continuous light reflecting on eyes. Also Saw et al., 2000 \& Saw et al., 2007), who found that a positive association between myopia and near-work activity such as reading and writing. In our study it was observed that many students did not like wear eyeglasses, it explained by Sala-i-Martin, Xavier, (2004) who stated that Approximately 10 percent of primary school age children in developing countries are thought to have vision problems, yet very few of them wear glasses.

\section{Conclusion}

\section{Based on the results of this study, it can be concluded that}

The common risk factor of R E among primary and preparatory school children are source of pollution inside and outside home and students living in mixed residential areas have higher RE compared with the other residential areas. 
The main recommendations from this study are

- Development of national programme for early detection of visual impairment, involving both preschoolers and school age children.

- Establishment of a school screening programme and follow-up for RE and low vision involving both preschoolers and school children.

- Governmental support for providing low-cost spectacles and school support to encourage children to wear their glasses.

- Health education program for persons contact with school children either parents or teachers about refractive error. In addition, health education sessions for students and their parents are needed to increase students awareness about risk factors of RE.

\section{References}

1. Abd El-Twaab M., (2004): Assessment of social class scale, Assiut University, Faculty of education, department of psychology.

2. Adegbehingbe B., Oladehinde M., Majemgbasan T., Onakpoya O., \& Osagiede E., (2005): Screening of adolescents for eye diseases in Nigerian high schools. Ghana Med J, 39 (4): 138-42.

3. Afghani T., Vine H., Bhatti A., Qadir M., Akhtar J., Tehzib M., \& Al-Shifa A., (2003): (ASAN)Refractive error study of one million school age children. Pakistan J Ophthalmol Oct; 19 (4): 101-7.

4. Alam H., Irfanullah Siddiqui M., Imtiaz S., \& Jafri A., (2008): Prevalence of refractive error in school children of Karachi. J Pak Med Assoc , 58(6):294-298.

5. Ali A., Ahmad I., \& Ayub S., (2007): Prevalence of undetected refractive errors among school children. of Lahore. Biomedica., 23(6): 96-101.

6. Allensworth D., Lawson E., Nicholson L., \& Wycho J., (1997): Schools and health: Our nation's investment. Committee on Comprehensive School Health Programs in Grades K-12 Division of Health Sciences Policy.

7. Ayanniyi A., Adepoju F., Ayanniyi R., \& Morgan R., (2010): Challenges, Attitudes and Practices of the Spectacle Wearers in a Resource- Limited Economy. Middle East Afr J Ophthalmol; 17:83-7.

8. Ayub Ali, Imran Ahmad \& Saima Ayub (2007): Prevalence of Undetected Refractive Errors among School Children, E:/Biomedica Vol.23 Jul. - Dec. 2007/Bio-21 (A).

9. Baltussen R., Naus J., \& Limburg H., (2008): Cost-effectiveness of screening and correcting refractive errors in school children in Africa, Asia, America \& Europe. Health Policy;89:20115.

10. Bataineh H., \& Khatatbeh A., (2008): Prevalence of refractive errors in school children (12-17 years of Tafila City. Sudanese J Public Health, 33(1):86-88.

11. Beers M., \& Porter R., (2006): Refractive error. Global Therapeutic Soluations in Ophthalmology. www.quantel-medical.com, p. 881-5.

12. Burns, E., Brady A., Dunn, M., Starr, B., \& Blosser, G., (2009): Pediatric primary care , chapter 28 . Eye disorder, Approaches to diseases management. fourth edition, Lippincott Williams \& Wilkins, pp 584-685.

13. Dandona R., \& Dandona L., (2001): Socioeconomic status and blindness. British Journal of ophthalmology, 85:1484-8.

14. Dandona R., Dandona L., Srinivas M., Sahare P., Narsaiah S., Muñoz S., Pokharel G., \& Ellwein L., (2002): Refractive error in children in rural population in India. Invest Ophthalmo lVis Sci; 43:615-22.

15. Datta P., (2014): Pediatric Nursing. 4rd, Jaypee Brothers Medica; Publishers (p) LTD,New Delhi, London and Panama. Pp, 409-410.

16. El -Bayoumy B., \& Saad A., (2007): Prevalence of refractive error and low vision among school children in Cairo. Eastern Mediterranean health journal,13(3 ):575-9.

17. El-Moselhy E., Ammar M., Abd-Allah K., Ismail I., \& Abd-Allah E., (2005a): Epidemiological study of risky behavior and their relation with mental ill health among secondary school students in Cairo. Egypt $\mathbf{J}$ Hosp Med, 19: 1-17.

18. El-Moselhy E., El-Azab R., Khalifa H., AbdAllah E., Ebrahim A., El-Masry H., ElShorbgy T., \& Abo Seif H., (2005b): Epidemiological study of the childhood disabilities: A household survey in four Egyptian Governorates. Egypt J Hosp Med, 20: 66-82.

19. Essam A., El-Moselhy, Hosam S., Abo-Seif, Eman S., Abd Allah \& Ahmed A., Ghandor (2011): Prevalence, Risk Assessment and Impacts of Eye Diseases among School Children in Cairo, Egypt, Journal of American Science;7(1).

20. Hashim S., Tan H., Wan-Hazabbah W., \& Ibrahim M., (2008): Prevalence of refractive error in Malay primary school children in suburban area of Kota Bharu, Kelantan, Malaysia. Ann Acad Med Singapore , 37(11):940-6. 
21. Hockeberry M., \& Wilson D., (2007): Nursing care of infants and children, 8ed,chapt 24. Pp, 1010-1016.

22. Katz J., Tielsch J., \& Sommer A., (1997): Prevalence and risk factors for refractive errors in an adult inner city population. investigative ophthalmology and visual science, 38:334-40.

23. Khalaj M., Gasemi M., \& Zeidi I., (2009): Prevalence of Refractive Errors in Primary School Children [7-15 Years] of Qazvin City. Eur J Sci Res;28:174-85.

24. 24.Khandekar R., \& Abdu-Helmi S., (2004): Magnitude and determinants of refractive error in Omani school children. Saudi Med J, 25 (10): 1388-93.

25. Kyle, T., (2009): Essentials of pediatric nursing of the child with a health disorder. First Indian preprint 2009, Lippincott Williams \& Wilkins, New York, Sydney. pp 1201-1204.PP 542-533.

26. 26.Kligman R., Stanton B., Geme J., Schor N., \& Beharman R., (2011): Nelson tectbook of pediatrics.Vision disorder $19^{\text {th }}$ chapter 613.Elserver sunders ,Philadelphia. Pp, 2152-2187.

27. Lempert P., (2004): The axial length/disc area ratio in anisometropic hyperopic amblyopia: a hypothesis for decreased unilateral vision associated with hyperopic anisometropia. Ophthalmology. Feb; 111 (2): 304-8.

28. Matta S., Matta P., Gupta V., \& Dev A., (2005): Refractive errors among adolescents attending Ophthalmic OPD. Ind Joun Comm Medicine. 04 - 2005-06; 31(2).

29. Murthy G., Gupta S., Ellwein L., Muñoz S., Pokharel G., Sanga L., \& Bachani D (2002):

Refractive error in children in an urban population in New Delhi. Invest Ophthalmol Vis Sci; 43:623-31.

30. Mutti D., Mitchell G., Moeschberger M., Jones L., \& Zadnik K., (2002): Parental myopia, near work, school achievement, and children's refractive error. Invest Ophthalmol Vis Sci. Dec; 43 (12): 3633-40.

31. Morgan I., Ohno-Matsui K., \& Saw S., (2012): Myopia. Lancet 379: 1739-1748. doi: 10.1016/s0140-6736(12)60272-4.

32. Ovenseri-Ogbomo, G., \& Assien, R., (2010): Refractive error in school children in Agona Swedru, Ghana.South African Journal of Optometry, 69(2), 86-92.

33. Padhye A., Khandekar R., Dharmadhikari S., Dole K., Gogate P., \& Deshpande M., (2010): Prevalence of uncorrected refractive error and other eye problems among urban and rural school children. Middle East Afr J Ophthalmol [serial online] 2009 [cited, Jun21];16:69-74. Available

from: http://www.meajo.org/text.asp?2009/16/2/69/538 64.

34. Pascolini D., \& Mariotti S., (2010): Global estimates of visual impairment: 2010. Br J Ophthalmol;96:614-8.

35. Pavithra M., Maheshwaran R., \& Rani Sujatha M., (2013) : Study on The Prevelance of Refractiv e Errors Among School Children of 7-1 5 Years Age Group in The Field Ractice Areas of A Medical College in Banglore.

36. Pan C., Ikram M., Cheung C., Choi H., Cheung C., (2013): Refractive errors and agerelated macular degeneration: a systematic review and meta-analysis. Ophthalmology 120: 2058-2065. doi: 10.1016/j.ophtha.2013.03.028

37. Resnikoff S., Pascolini D., Mariotti S., \& Pokharel G., (2008): Global magnitude of visual impairment caused by uncorrected refractive errors in 2004. Bull World Health Organ; 86:63-70.

38. Rahi J., Canberland P., \& Peckham C., (2010): Improving detection of blindnessin childhood:The British childhood vision Improvement study, Pediatrics126:e895-e903.

39. Rose K., Morgan I., Ip J., Kifley A., Huynh S., Smith W., \& Mitchell P., (2008): Ophthalmology-Outdoor activity reduces the prevalence of myopia in children. $\mathrm{j}$. ophtha , 115(8):1279-1285.

40. Rose, K., Morgan, I., Smith, W., Burlutsky, G., Mitchell, P., \& Saw, S., (2008): Myopia, lifestyle, and schooling in students of Chinese ethnicity in Singapore and Sydney. Achieve of Ophthalmology, 126, 527-30. http://dx.doi.org/10.1001/archopht.126.4.527.

41. Ricci S., \& kyle, T., (2009): Maternity and Pediatric Nursing, chapter ; nursing care of the child with a disorder of the eyes or ears. Textbook Wolter Kluwer Health - Lippincott Williams \& Wilkins pp 1201-1204.

42. Saw S., Zhang M., Hong R., Fu Z., Pang M., \& Tan D., (2002): Near-work activity, nightlights, and myopia in the Singapore-China study. Arch Ophthalmol. May; 120 (5): 620-7.

43. Saw S., Cheng A., Fong A., Gazzard G., Tan D., et al., (2007): School grades \& myopia. Ophthalmic Physiol Opt. Mar;27(2):126-9. [PubMed].

44. Sala-i-Martin, Xavier, Gernot Doppelhofer \& Ronald Miller. (2004): Determinants of LongTerm Growth: A Bayesian Averaging of Classical Estimates (BACE) Approach,\| American Economic Review. 94(4): 813-835.

45. Sharma S., Vashisht B., Kalhan M., \& Goel M., (2009): Magnitude of Refractive Errors among school children in a rural block of 
Haryana. The Internet Journal of Epidemiology;6(2). DOI: 10.5580/1e5f.

46. Shrestha R., Toshi M., Ghisingh R., Pradhan P., Shahya S., \& Rizyal A., (2009): Ocular morbidity among children studying in private school of Kathmandu Valley: A prospective cross-sectional study. J Nepal Med College, 8: 43-6.

47. Shukrallah A., Mostafa H., \& Magdi S., (1997): The current state of the disability question in Egypt. Preliminary national study. North-South Inserm Network. Retrieved from: http://www.ahedegypt.org/pubs/CSODQE/CSO DQE_001.pdf.

48. Tay M., AuEong K., \& Lim M., (1992): Myopic \& educational attainment in 421116 young Singaporean males. Ann Acad Med, Singapore; 21:785-91.

49. Vitale, S., Cotch, M., \& Sperduto, R., (2006): Prevalence of visual impairment in the United States. JAMA 295, 2158-63.

50. Vivek T., Sandip Z., Janardhan V., \& Tapasi P., (2008): Prevalence of refractive errors in children aged 7-15 years in Gujarat, India. (cited, Sept 15). Available from: URL: http://www.nhlmmcgym.com/indianjournal6.htm.

51. Wong T., (2007): Variation in ocular biometry in an adult Chinese population in Singapore: The Tanjongpagar survey. investigative ophthalmology and visual science,42:73-8o.

52. WHO's global initiative (Vision 2013): http://www.vision2020.org/main.cfm?type=WH ATVISION2020

WHO Disease and injury country estimates. Retrieved Nov 11, 2009.9.

53. Yingyong P., (2010): "Risk factors for refractive errors in primary school children (6-12 years old) in Nakhon Pathom Province". J. Med. Assoc. Thai., 93(11): 1288-1293.

54. Zhao J., Mao, J., Luo, R., Li, F., Pokharel, G., \& Ellwein, L., (2004): Accuracy of non cycloplegic auto refraction in school-age children in China. Optometry \& Vision Science, 81,49-55http://dx.doi.org/10.1097/00006324200401000-00010. 